$\xi=-1$

\title{
Diametral Paths in Total Graphs of Paths, Cycles and Stars
}

\author{
T. A. Mangam ${ }^{1}$, J. V. Kureethara ${ }^{1} *$ \\ ${ }^{1}$ CHRIST (Deemed to be University), Hosur Road, Bangalore, Karnataka, India - 560029 \\ *Corresponding author E-mail: frjoseph@christuniversity.in
}

\begin{abstract}
The diametral path of a graph is the shortest path between two vertices which has length equal to diameter of that graph. Total graph of a graph is a graph that has vertices representing all vertices and edges of the original graph and edges representing every vertex-vertex adjacency, edge-edge adjacency and edge-vertex incidence. In this paper, the number of diametral paths is determined for the paths, cycles and stars and their total graphs.
\end{abstract}

Keywords: Diametral path, Path, Cycle, Star, Diameter, Total graph

\section{Introduction}

Outliers provide very interesting and sometimes vital information in any system. In a graph, peripheral vertices are some types of outliers associated with its structure itself. Peripheral vertices are vital in identifying the diametral paths of a graph. Deogun and Kratsch introduced and discussed the concept of diametral paths. [3]

Recently, this authors have initiated the studies on diametral paths in total graphs.[4,5] Total graph of a graph is a graph that has vertices representing all vertices and edges of the original graph and edges representing every vertex-vertex adjacency, edge-edge adjacency and edge-vertex incidence. Hence, the number of edges in the total graph $\mathrm{T}(\mathrm{G})$ of a connected graph $\mathrm{G}$ is

$\frac{1}{2} \sum_{v \in V(G)} d(v)(d(v)+2) \cdot[4]$

It is proved that for a graph $G$, the diameter of $T(G)$, denoted by $\operatorname{diam}(\mathrm{T}(\mathrm{G}))$ is equal to $\operatorname{diam}(\mathrm{G})$ or $\operatorname{diam}(\mathrm{G})+1 .[4]$ The diametral paths in total graphs of Complete Graphs, Complete Bipartite Graphs and Wheels are studied in [5].

We now discuss on the number of diametral paths in paths, cycles and stars, and their total graphs. For more about graphs and various concepts in graphs that are not explained explicitly in this paper, refer to [1] and [6].

\section{Paths}

A path graph is a connected graph with uninterrupted sequence of distinct vertices and edges. A path with $n$ vertices is denoted by $P_{n}$. Since $\operatorname{diam}\left(P_{n}\right)=n-1$, there is only one diametral path in $P_{n}$ which is the path itself with peripheral vertices as its endvertices. It can be noted that $\operatorname{diam}\left(\mathrm{T}\left(\mathrm{P}_{\mathrm{n}}\right)\right)=\mathrm{n}-1$. There are four peripheral vertices in $T\left(P_{n}\right)$ which are represented by the endvertices and pendant edges of $\mathrm{P}_{\mathrm{n}}$. We now have the number of diametral paths of the total graph of a path in the next result.

Theorem 1The number of diametral paths of $T\left(P_{n}\right)$ is $2 n-1$.
Proof Consider a $P_{n}$ with vertices $v_{1}, v_{2}, \ldots, v_{n}$ and edges $e_{1}, e_{2}$ ... $e_{n-1}$ such that each $e_{i}$ is incident on $v_{i}$ and $v_{i+1}$ for $1 \leq \mathrm{i} \leq \mathrm{n}-1$. Since $\operatorname{diam}\left(\mathrm{T}\left(\mathrm{P}_{\mathrm{n}}\right)\right)$ is $\mathrm{n}-1$, any diametral path in $\mathrm{T}\left(\mathrm{P}_{\mathrm{n}}\right)$ will have one of the following pairs of vertices as end vertices.

$$
\begin{aligned}
& \text { (1) } v_{1} \text { and } v_{n} \\
& \text { (2) } v_{1} \text { and } e_{n-1} \\
& \text { (3) } e_{1} \text { and } v_{n}
\end{aligned}
$$

In case (1), there is exactly one diametral path between $v_{1}$ and $v_{n}$ in $\mathrm{T}\left(\mathrm{P}_{\mathrm{n}}\right)$.

In case (2), there are n-1 diametral paths which are $\mathrm{v}_{1} \mathrm{v}_{2} \ldots \mathrm{v}_{\mathrm{k}} \mathrm{e}_{\mathrm{k}} \ldots$ $\mathrm{e}_{\mathrm{n}-1}$ where $1 \leq \mathrm{k} \leq \mathrm{n}-1$.

In case (3), there are $n-1$ diametral paths which are $\mathrm{e}_{1} \mathrm{e}_{2} \ldots$ $\mathrm{e}_{\mathrm{k}} \mathrm{v}_{\mathrm{k}+1} \mathrm{v}_{\mathrm{n}}$ where $1 \leq \mathrm{k} \leq \mathrm{n}-1$.

Hence total number of diametral paths in $T\left(P_{n}\right)=1+n-1+n-1=2 n-1$. We now move to the case of cycle graphs.

\section{Cycles}

A cycle graph is nothing but a path graph with the same initial and terminal vertices.

It can be noted that $\operatorname{diam}\left(\mathrm{C}_{\mathrm{n}}\right)=\mathrm{d}=[\mathrm{n} / 2\rfloor$ and $\operatorname{diam}\left(\mathrm{T}\left(\mathrm{C}_{\mathrm{n}}\right)\right)=[\mathrm{n} / 2\rceil$. The number of diametral paths is determined in cycles and their total graphs.

Lemma 1 A cycle $C_{n}$ has n diametral paths.

Proof Diameter of a cycle $C_{n}$ is given by $\operatorname{diam}\left(C_{n}\right)=d=\lfloor n / 2\rfloor$. Let $v_{1}, v_{2} \ldots v_{n}$ be the $n$ vertices of $C_{n}$. Considering the diametral paths from every vertex $v_{i}$ through $v_{i+1}, 1 \leq i \leq n-1$ and one diametral path from $v_{n}$ through $v_{1}$, there are $n$ diametral paths. Hence cycle $C_{n}$ has n diametral paths.

Theorem 2 The number of diametral paths in $T\left(C_{n}\right)$ is $n(n+1)$ when $n$ is odd and $n(n+2)$ when $n$ is even.

Proof We complete the proof by analysing two cases.

A. Consider $\mathrm{n}$ to be odd. 
Let $\mathrm{v}_{1}, \mathrm{v}_{2} \ldots \mathrm{v}_{\mathrm{n}}$ be the vertices and $\mathrm{e}_{1}, \mathrm{e}_{2} \ldots \mathrm{e}_{\mathrm{n}}$ be the corresponding edges of $C_{n}$ such that each $e_{i}$ is incident on $v_{i}$ and $v_{i+1}$ for $1 \leq i \leq$ $\mathrm{n}-1$ and $\mathrm{e}_{\mathrm{n}}$ incident on $\mathrm{v}_{\mathrm{n}}$ and $\mathrm{v}_{1}$. Hence $\mathrm{T}\left(\mathrm{C}_{\mathrm{n}}\right)$ has vertices $\mathrm{v}_{1}, \mathrm{v}_{2} \ldots$ $\mathrm{v}_{\mathrm{n}}$ and new vertices $\mathrm{e}_{1}, \mathrm{e}_{2} \ldots \mathrm{e}_{\mathrm{n}}$. When $\mathrm{n}$ is odd, $\mathrm{T}\left(\mathrm{C}_{\mathrm{n}}\right)$ has a diametral path from every $v_{i}$ to a specific $e_{j}$. Since $\operatorname{diam}\left(T\left(C_{n}\right)\right)=$ $\mathrm{d}=[\mathrm{n} / 2]$, every diametral path has one endvertex as $\mathrm{v}_{\mathrm{i}}$ and the other endvertex $\mathrm{e}_{(\mathrm{i}+\mathrm{d}-1)}$ for $1 \leq \mathrm{i} \leq(\mathrm{n}-\mathrm{d}+1)$ and $\mathrm{e}_{(\mathrm{i}-\mathrm{d})}$ for the remaining $i$. The diametral path from $\mathrm{v}_{\mathrm{i}}$ to $\mathrm{e}_{(\mathrm{i}+\mathrm{d}-1)}$ or $\mathrm{e}_{(\mathrm{i}-\mathrm{d})}$ accordingly satisfies one of the following conditions.

[(1)] It can pass through only $v_{i}$ 's.

[(2)] It can pass through only $\mathrm{e}_{i}$ 's.

[(3)] It can pass through $v_{i}$ 's to some stage and $e_{i}^{\prime}$ s from

there.

In case (1), since each vertex $v_{i}$ has two neighbors in $v_{1}, v_{2} \ldots v_{n}$, there are two diametral paths through only $v_{j}$ 's, from each $v_{i}$.

In case (2), since each vertex $v_{i}$ has two neighbors in $e_{1}, e_{2} \ldots e_{n}$, there are two diametral paths through only $e_{i}$ 's, from each $v_{i}$.

In case (3), there are $n-3 v_{j}$ 's left through which the diametral path can pass when the initial vertex $v_{j}$ and neighbors of the corresponding $e_{j}$ are excluded. Hence there are n-3 diametral paths in this case from each $\mathrm{v}_{\mathrm{i}}$.

Since there are $\mathrm{n}$ vertices, the number of diametral paths $=n(2+2+n-3)=n(n+1)$.

\section{B. Consider $\mathrm{n}$ to be even.}

Let $\mathrm{v}_{1}, \mathrm{v}_{2} \ldots \mathrm{v}_{\mathrm{n}}$ be the vertices and $\mathrm{e}_{1}, \mathrm{e}_{2} \ldots \mathrm{e}_{\mathrm{n}}$ be the corresponding edges of $C_{n}$ such that each $e_{i}$ is incident on $v_{i}$ and $v_{i+1}$ for $1 \leq i \leq n$ 1 and $e_{n}$ incident on $v_{n}$ and $v_{1}$. Hence $T\left(C_{n}\right)$ has vertices $v_{1}, v_{2} \ldots$ $v_{n}$ and new vertices $e_{1}, e_{2} \ldots e_{n}$. Since $\operatorname{diam}\left(T\left(C_{n}\right)\right)=\lceil n / 2\rceil, T\left(C_{n}\right)$ has diametral paths from every $v_{i}$ to a specific $v_{j}, e_{j}$ and $e_{(j-1)}$ and diametral paths with endvertices $e_{i}$ 's. Since every diametral path with one endvertex as $v_{i}$ has the other endvertex from one of the following categories.

[(a)] $\mathrm{v}_{\mathrm{i}+\mathrm{d}}$ or $\mathrm{e}_{(\mathrm{i}+\mathrm{d}-1)}$ or $\mathrm{e}_{\mathrm{i}+\mathrm{d}}$ for $1 \leq \mathrm{i} \leq \mathrm{n}-\mathrm{d}$

[(b) $] \mathrm{v}_{1}$ or $\mathrm{e}_{1}$ or $\mathrm{e}_{\mathrm{n}}$ for $\mathrm{i}=\mathrm{d}+1$

[(c)] $\mathrm{v}_{\mathrm{i}-\mathrm{d}}$ or $\mathrm{e}_{\mathrm{i}-\mathrm{d}}$ or $\mathrm{e}_{(\mathrm{i}-\mathrm{d}-1)}$ for the remaining $\mathrm{i}$.

We consider diametral paths in three cases.

[(1)] The endvertices are $\mathrm{v}_{\mathrm{i}} \mathrm{s}$.

[(2)] One endvertex is $v_{i}$ and other is a specific $e_{j}$.

[(3)] The endvertices are $e_{i}$ 's.

In case (1), there is a diametral path from each $v_{i}$ only through vertices $v_{j}$ 's to a specific $v_{j}$. Since there are $n v_{i}$ 's, there are $n$ diametral paths in this case.

In case (2), there are two diametral paths from each $v_{i}$ to corresponding $\mathrm{e}_{\mathrm{j}}$ through vertices $\mathrm{v}_{\mathrm{k}}$ 's. Also there are two diametral paths from each $v_{i}$ to corresponding $e_{j}$ through $e_{k}$ 's. There are diametral paths which pass through $\mathrm{v}_{\mathrm{k}}$ 's to some stage and $\mathrm{e}_{\mathrm{k}}$ 's from there. Since there are $n-4$ vertices left of the $v_{i}$ 's when the initial vertex $v_{i}$ and neighbours of the corresponding $e_{j}$ s are excluded, there are n-4 diametral paths from each $v_{i}$. Since there are $n v_{i}$ 's, the number of diametral paths is $n(2+2+n-4)=n^{2}$.

In case (3), from each $\mathrm{e}_{\mathrm{i}}$, there is a diametral path only through vertices $e_{j}$ 's to a specific $e_{j}$.

Since there are $n e_{i}$ 's, there are $n$ diametral paths in this case.

Hence the total number of diametral paths $=n+n^{2}+n=n(n+2)$.

We now proceed with the discussions on stars.

\section{Stars}

A star graph is a connected graph with exactly one vertex is adjacent to every other vertex and no two of the other vertices are adjacent to each other. It can be noted that $\operatorname{diam}\left(T\left(\mathrm{~K}_{1, \mathrm{n}}\right)\right)=2$ for $\mathrm{n}$ $\geq 2$. The number of diametral paths is determined in stars and their total graphs.

Lemma 2 A star $K_{1, n},(n \geq 2)$ has ${ }^{n} C_{2}$ diametral paths.

Proof It is a known fact that $\operatorname{Diam}\left(\mathrm{K}_{1, \mathrm{n}}\right)=2$. Since there are $\mathrm{n}$ peripheral vertices, there are ${ }^{n} C_{2}$ diametral paths through the central vertex.

Hence the number of diametral paths $={ }^{\mathrm{n}} \mathrm{C}_{2}$.

Theorem 3 The number of diametral paths in $T\left(K_{1, n}\right)$ is $\left(\left(5 n^{2}\right)\right.$ $5 n) / 2$ for $n \geq 2$.

Let $\mathrm{v}_{1}, \mathrm{v}_{2} \ldots \mathrm{v}_{\mathrm{n}+1}$ be the vertices and $\mathrm{e}_{1}, \mathrm{e}_{2} \ldots \mathrm{e}_{\mathrm{n}}$ be the corresponding edges of $K_{1, n}$ such that each $e_{i}$ is incident on $v_{i}$ and $v_{n+1}$ for $1 \leq \mathrm{i}$ $\leq \mathrm{n}$. Hence $\mathrm{T}\left(\mathrm{K}_{1, \mathrm{n}}\right)$ has vertices $\mathrm{v}_{1}, \mathrm{v}_{2} \ldots \mathrm{v}_{\mathrm{n}+1}$ and new vertices $\mathrm{e}_{1}, \mathrm{e}_{2} \ldots \mathrm{e}_{\mathrm{n}}$.

Since $\operatorname{diam}\left(\mathrm{T}\left(\mathrm{K}_{1, \mathrm{n}}\right)\right)=2$, the peripheral vertices are $\mathrm{v}_{1}, \mathrm{v}_{2} \ldots \mathrm{v}_{\mathrm{n}}$, $\mathrm{e}_{1}, \mathrm{e}_{2} \ldots \mathrm{e}_{\mathrm{n}}$. Hence $\mathrm{T}\left(\mathrm{K}_{1, \mathrm{n}}\right)$ has diametral paths with one endvertex to be $v_{i}$ and the other endvertex to be $v_{j}$ or $e_{j}(i \neq j)$. Since there are $\mathrm{n}$ peripheral vertices $\mathrm{v}_{1}, \mathrm{v}_{2} \ldots \mathrm{v}_{\mathrm{n}}$, the number of diametral paths through $v_{n+1}$ with endvertices $v_{i}$ and $v_{j}(i \neq j)$ is ${ }^{n} C_{2}$. Also a diametral path with endvertices $v_{i}$ and $e_{-}\{j\}(i \neq j)$ passes through $v_{n+1}$ or $e_{i}$. Since diametral path from each $v_{i}$ can have $n-1$ possible endvertices $\mathrm{e}_{1}, \mathrm{e}_{2} \ldots \mathrm{e}_{\mathrm{i}-1}, \mathrm{e}_{\mathrm{i}+1} \ldots \mathrm{e}_{\mathrm{n}}$, there are $\mathrm{n}-1$ diametral paths through $\mathrm{v}_{\mathrm{n}+1}$ and $\mathrm{n}-1$ diametral paths through $\mathrm{e}_{\mathrm{i}}$.

Since there are $n$ such $v_{i}$ 's, the number of diametral paths is $n(n-$ $1+n-1)=n(2 n-2)$.

Hence total number of diametral paths $={ }^{\mathrm{n}} \mathrm{C}_{2}+\mathrm{n}(2 \mathrm{n}-2)=\left(\left(5 \mathrm{n}^{2}\right)\right.$ $5 n) / 2$.

\section{Conclusion}

The number of diametral paths is determined for paths, stars and cycles and their total graphs are computed here. The focus of further research would be the decomposition or packing of total graphs of paths, cycles and starts into diametral paths.

\section{References}

[1] Buckley F \& Harary F (1990), Distance in Graphs, Perseus Books, New York

[2] Buckley F \& Lewinter M (1993), Graphs with all diametral paths through distant central vertices, Mathematical and Computer Modelling 17, 11, 35-41.

[3] Deogun JS \& Kratsch D (1995), Diametral path graphs. In: Nagl, M (eds.) Graph-Theoretic Concepts in Computer Science, LNCS, 1017 Springer, Berlin, Heidelberg, 344-357.

[4] Mangam TA \& Kureethara JV (2017), Diametral Paths in Total Graphs, International Journal of Pure Applied Mathematics 117, 12 273-280.

[5] Mangam TA \& Kureethara JV (2017), Diametral Paths in Total Graphs of Complete Graphs, Complete Bipartite Graphs and Wheels, International Journal of Civil Engineering and Technology $8,5,1212-1219$.

[6] Ore O (1968), Diameters in Graphs, Journal of Combinatorial Theory, $5,1,75-81$. 